\title{
The Algebraic Structure of Quantity Calculus
}

\author{
Álvaro P. Raposo ${ }^{1}$ \\ ${ }^{1}$ Department of Applied Mathematics, Universidad Politécnica de Madrid, Av. Juan de Herrera, 6, 28040, Madrid, Spain, \\ alvaro.p.raposo@upm.es
}

The algebraic structure underlying the quantity calculus is defined axiomatically as an algebraic fiber bundle, that is, a base structure which is a free Abelian group together with fibers which are one dimensional vector spaces, all of them bound by algebraic restrictions. Subspaces, tensor product, and quotient spaces are considered, as well as homomorphisms to end with a classification theorem of these structures. The new structure provides an axiomatic foundation of quantity calculus which is centered on the concept of dimension, rather than on the concept of unit, which is regarded as secondary, and uses only integer exponents of the dimensions.

Keywords: Quantity calculus, algebraic structure, group of dimensions.

\section{INTRODUCTION}

The foundations of quantity calculus have been the subject of research for more than a century, and recent papers as those by Kitano [1], Krystek [2], Atkey et al. [3] or Domotor [4] show that its formalization is not completely settled yet. Moreover, in the last years many authors are calling for a discussion of fundamental concepts regarding quantity calculus $[5,6,7,8,9]$. In fact, the first two papers mentioned represent two different visions on the subject. The paper by Kitano assumes a structure of quantity calculus centered in the definition of units, upon which the rest of the structure is built. In contrast, the paper by Krystek recovers the call of some authors in past decades to locate the concept of dimension in the center of the scene. The latter view is what the widespread use of dimensional analysis (see, e.g., $[10,11]$ ) suggests that the structure of quantity calculus should be, but the fact is that there is not a well defined algebraic structure supporting it. The goal of this paper is to provide such a structure. On another basis, the paper by Domotor deserves a separate comment, for it dives into ontological discussion of the relationship between quantities and their values and provides an algebraic tool to describe it by means of torsors, which is further commented below.

By quantity calculus it is understood the algebra of the operations performed between physical quantities, which are three: product of quantities, product of a number times a quantity and addition of quantities of the same kind. It is customary to write a quantity $q$ as the product of a number times a unit which, in the present notation of the International Vocabulary of Metrology (VIM) [12], is denoted as

$$
q=\{q\}[q]
$$

where $[q]$ stands for the unit and $\{q\}$ is the number of times $q$ comprises that unit. Notice that, as the VIM explicitly warns in its Note 5 under the definition of quantity, this concept is that of a scalar quantity, and the way to consider vector or tensor quantities is through their components which accommodate to this definition. The operations are then performed as

$$
\begin{aligned}
q_{1} q_{2} & =\left(\left\{q_{1}\right\}\left\{q_{2}\right\}\right)\left(\left[q_{1}\right]\left[q_{2}\right]\right), \\
\alpha q & =(\alpha\{q\})[q], \\
q_{1}+q_{2} & =\left(\left\{q_{1}\right\}+\left\{q_{2}\right\}\right)\left[q_{1}\right],
\end{aligned}
$$

where $\alpha$ is a number, $q, q_{1}$, and $q_{2}$ are quantities and, in the last equation, $\left[q_{1}\right]=\left[q_{2}\right]$ is assumed. Although this way of performing the operations is standard, the algebraic structure to which these three operations give rise is still under study, and it is the particular form of equation (2c) what makes it quite different from other usual structures such as rings, vector spaces or algebras.

The history of quantity calculus, in the words of Domotor [4], is the story of the search for the intrinsic algebraic structures underlying dimensional analysis, measurement units and measurement uncertainty of quantity values. This history is unsually long, and the reader is referred to the excellent review by de Boer [13] for a detailed account. However, a few landmarks are worth mentioning in order to situate the contribution of the present paper. Its origin goes back to Fourier in 1822 [14], when he introduced the idea of dimension of a quantity and the concept of homogeneity of dimensions in a valid physical equation, thus initiating the view centered on dimensions. Contemporarily, Gauss, in 1832 [15], proposed the first so called absolute system of 
units, relying on the units for length, mass and time, which eventually became the cgs system. Another step is due to Maxwell in 1873 [16], when he stated that the expression of a quantity has two factors or components, as in equation (1); in fact, the present notation in that equation comes from that of Maxwell. In 1914 Buckingham published his celebrated Pi Theorem [17], although there was not a solid framework on which to base its proof. For the remaining duration of the 20th century there is a quest for the formalism underlying the quantity calculus. According to de Boer, Wallot was the first to claim the concept of quantity as central, and not just as the product of a number and a unit. Then Landolt [18], in 1945, made the first attemp to give an axiomatic foundation, with the important step of recognizing the group properties of the operations between quantities. In the $1950 \mathrm{~s}$ Fleischmann [19], also according to de Boer, took another step forward by distinguishing the quantities from the kinds of quantities, the latter exhibiting the structure of a group. In the following years several authors contributed to set up a description of the algebraic structure but, curiously enough, two divergent paths were followed. On the one hand authors such as Fleischmann, Quade [20] or de Boer himself called for a description centered on the quantities and the concept of dimension. On the other hand, authors such as Drobot [21], Whitney [22], and Carlson [23] developed an algebraic structure which resembled that of quantity calculus, but was centered on the concept of unit over which the rest of the set of quantities was built. The recent paper by Kitano [1] can be seen as the zenith of this viewpoint, for he introduces a theory of comparison of the different systems of units which provides new insight into the subject. In a rough summary, this structure starts with a system of units $\left\{u_{1}, \ldots, u_{n}\right\}$ and writes any quantity $q$ in a unique way as

$$
q=\alpha u_{1}^{r_{1}} \cdots u_{n}^{r_{n}}
$$

where $\alpha$ is a real number and $r_{1}, \ldots, r_{n}$ are rational numbers. Therefore, the algebraic structure depicted by this theory is $\mathbb{R} \times \mathbb{Q}^{n}$, where the factor $\mathbb{R}$ hosts the numerical value of $q$ relative to this system of units, while $\mathbb{Q}^{n}$ hosts the rational exponents of the units and exhibits a linear space structure. However, the rules (not the algebraic properties) to perform the three operations need to be set in the axioms, which is not quite acceptable in an axiomatic description of an algebraic structure. As a consequence of the central role of the unit systems, we can find quantities which, dependending on the unit system of choice, can be compared or cannot, can be added or cannot.

Notwithstanding its merits, the accepted description of the algebraic structure of quantity calculus has two drawbacks that need to be addressed. Firstly, as said before, it relies on the concept of unit as central. This is not satisfactory since units are the result of an arbitrary agreement and, thus, can be easily changed, while the concept of dimension is more resilient. (The arbitrariness, of course, is only from the algebraic viewpoint; in fact, the choice of base units in a sys- tem of units, say the SI, is far from arbitrary, for they need to be as constant and accurate as possible and reproducible). The history of the units in electromagnetism at the end of the 19th century and beginning of the 20th shows it clearly: the mess of different systems of units, all of them trying to describe electromagnetic quantities in a purely mechanical context, was definitely clarified with the proposal of Giorgi [24] to introduce a separate unit for one of the electromagnetic quantities, that is, introducing a new and independent dimension.

Secondly, the use of fractional exponents in the units. It is a remarkable fact that, albeit the possibilities allowed by Bridgman's theorem on dimensional analysis [25], dimensionful physical quantities enter into physically valid equations only through the three operations mentioned at the beginning. Therefore, only integer exponents of quantities, and thus of units, should be expected. Although the use of fractional exponents is widespread, we claim that it is not necessary. Several examples come to mind, as the case of square roots of quantities (the use of Pythagoras' theorem, the period of a pendulum, the standard deviation of a random quantity...) but in all cases the square root acts on a quantity which is already a square. An algebraic structure for quantity calculus, which allows fractional exponents, is oversized.

It is noticeable that Quade [20], following Fleischmann, developed a description of the algebraic structure entirely with integer exponents of the units instead of fractional ones, and also tried to center it on the concept of dimension. Unfortunately, this approach, which pointed in the correct direction, was superseded by the one described above. The cause of moving the focus from dimensions to units is, probably, the absence of a known algebraic structure which fits exactly the operations between quantities. But, fortunately, the recent paper by Krystek [2] resumes the quest for this structure.

Drawing from the ideas of Quade and Krystek, the goal of this paper is to introduce a new algebraic structure, based on a simple set of axioms, which accounts exactly for the properties of quantity calculus and overcomes the two aforementioned problems. The structure is centered in the group of dimensions and the quantities are placed upon it gathered in fibers over the dimensions. Hence, the structure can be described as an algebraic fiber bundle. The axioms allow to define systems of units and, as a consequence, but not as a definition, to write quantities as in equation (1). Then the properties of the maps $\{\cdot\}$ and $[\cdot]$ are studied and the validity of equations (2) is established (not postulated, as usual). These are the contents of section 2. Section 3 shows how to construct new spaces of quantities from old ones as subspaces or by means of tensor products or quotients. The quotient of a space of quantities is seen to be the tool to reduce dimensions as it is usually done, for instance, to get natural units. In section 4 the tool for the comparison is defined and studied: the homomorphism of spaces of quantities, which allows us to characterize and classify the spaces. This classification is compared with that of Kitano in a final section of conclusions and also a comment on Domotor's torsor theory is considered. 


\section{GROUP OF DIMENSIONS AND SPACE OF QUANTITIES}

Our object of study is a set $Q$ of quantities and the operations defined within it, which is referred to as a space of quantities. The elements of $Q$ will be denoted by lowercase latin letters, particularly $q, r, s$. These quantities, as it is detailed below, can be multiplied and added among them and also multiplied by scalar numbers from a field $F$, whose elements will be denoted by lowercase Greek letters, particularly $\alpha, \beta$.

Example 2.1. For further reference, we assign symbols to the following spaces of quantities: $Q_{\mathrm{geom}}$, the space of quantities of geometry, that is, all the quantities needed to deal with lengths, areas, volumes, angles, etc. $Q_{\text {time, }}$ the space of quantities to measure time. $Q_{\mathrm{kin}}$, the space of quantities of kinematics. $Q_{\mathrm{mech}}$, the space of quantities of mechanics. $Q_{\mathrm{phys}}$, the space of quantities of physics.

\subsection{Group of dimensions}

As noticed in the introduction, main role in the structure is played by the dimension of a quantity. Under this viewpoint, the dimension is an intrinsic property of a quantity, in contrast to its numerical value, which depends on the unit chosen, or the unit itself, which can be changed arbitrarily. Therefore, each quantity must have a firm link with its dimension in the present scheme better than a link with a unit or its numerical value with respect to that unit, despite the latter being what equation (1) suggests. To that end, let us first define properly the set of dimensions. The properties which characterize this set have been well described in the paper by Krystek [2], and they are just summarized here: dimensions can be multiplied and show the structure of an Abelian group with two further properties which characterize this group. First, no element is torsion, for there is no dimensionful quantity which multiplied by itself finitely many times becomes a quantity of dimension one. Second, it is finitely generated. Therefore, we adopt the following definition.

Definition 2.2. A group of dimensions is a finitely generated free Abelian group.

In this paper such a group is generally denoted by $\mathscr{D}$ and its elements by uppercase letters in roman sans-serif type such as $A, B \ldots$ (as stated in the VIM). The identity element of the group, denoted $1_{\mathscr{D}}$, is the dimension of the so called dimensionless quantities (quantities of dimension one are preferred).

Two properties of finitely generated free Abelian groups (or, equivalenty, free $\mathbb{Z}$-modules) are of interest to us [26]. In the first place, there exists the concept of basis: an independent (finite) set of generators. If $\left\{A_{1}, \ldots, A_{k}\right\}$ is such a basis for a group $\mathscr{D}$ then any element $B$ has a unique expression in terms of the form $B=A_{1}^{n_{1}} \cdots A_{k}^{n_{k}}$, where the exponents $n_{1}, \ldots, n_{k}$ are integer numbers. The number $k$ of generators of any basis is called the rank of the group, and is a characteristic property of it. In the second place, such a group is isomorphic with the direct product of $k$ infinite cyclic groups: $\mathscr{D} \cong\left\langle\mathrm{A}_{1}\right\rangle \times \cdots \times\left\langle\mathrm{A}_{k}\right\rangle$, where $\left\langle\mathrm{A}_{i}\right\rangle=\left\{\mathrm{A}_{i}^{n}: n \in \mathbb{Z}\right\}$.
Example 2.3. The groups of dimensions of the systems of quantities given in example 2.1 are, respectively, the following: $\mathscr{D}_{\text {geom }}=\langle\mathrm{L}\rangle$, the free Abelian group generated by $\mathrm{L}$, which denotes length. $\mathscr{D}_{\text {time }}=\langle\mathrm{T}\rangle$, generated by $\mathrm{T}$ (time). $\mathscr{D}_{\text {kin }}=\langle\mathrm{L}, \mathrm{T}\rangle$, generated by $\mathrm{L}$ and $\mathrm{T} . \mathscr{D}_{\text {mech }}=\langle\mathrm{L}, \mathrm{T}, \mathrm{M}\rangle$, generated by $\mathrm{L}, \mathrm{T}$ and $\mathrm{M}$ (mass). $\mathscr{D}_{\text {phys }}=\langle\mathrm{L}, \mathrm{T}, \mathrm{M}, \mathrm{I}, \Theta\rangle$, generated by $\mathrm{L}, \mathrm{T}, \mathrm{M}, \mathrm{I}$ (electric current) and $\Theta$ (temperature).

\subsection{Space of quantities}

The link between a quantity and its dimension is made by means of a projection map $\operatorname{dim}: Q \rightarrow \mathscr{D}$. This map is a surjection.

Example 2.4. If $h$ is Planck's constant then $\operatorname{dim}(h)=$ $\mathrm{L}^{2} \mathrm{~T}^{-1} \mathrm{M}$; and $\theta$, the angle at a vertex of a triangle, yields $\operatorname{dim}(\theta)=1_{\mathscr{D}}$; each one in the appropriate setting.

In order to reflect that the dimension of a product of quantities is the product of the dimensions of the quantities the projection map must be a homomorphism with respect to the product of quantities.

In this way we follow the general concept studied by Atkey et al. [3] of a fiber bundle approach to quantities and dimensions. All the quantities with the same dimension, say A, form a set called a fiber, for it can be written as the inverse image of that dimension: $\operatorname{dim}^{-1}(A)$. As the VIM explicitly states, quantities of the same kind belong to the same fiber, while the opposite is not necessarily true. However, the algebraic structure cannot distinguish this detail. In each fiber quantities can be added and multiplied by scalars in a field $F$, resulting in quantities of the same dimension. These operations give the fiber the structure of a vector space over the field $F$. Moreover, since the comparison of each quantity in the fiber with a reference in the fiber, the unit, yields a single number, as in equation (1), that vector space is one dimensional (the latter in the sense of vector space dimension over $F$ ). There are some quantities which are intrinsically positive (mass, absolute temperature) and, thus, a full linear space seems to be oversized for them. However, differences of these quantities must also be considered in the framework. Since the algebraic structure cannot distinguish if a quantity is an absolute temperature or a difference of temperatures, the full linear space structure has to be allowed.

The field $F$ is usually assumed to be that of the real numbers but so far there is no algebraic reason to restrict the definition to it. We are now ready to give an axiomatic definition of a space of quantities which takes into account all the aforementioned elements.

Definition 2.5. A space of quantities with group of dimensions $\mathscr{D}$ over the field $F$ is a set $Q$, together with a surjective map dim: $Q \rightarrow \mathscr{D}$ such that:

(i) for each $\mathrm{A} \in \mathscr{D}$, the fiber $\operatorname{dim}^{-1}(\mathrm{~A})$ has the structure of a one dimensional vector space over $F$, 
(ii) there is a product defined in $Q$ which makes it into an Abelian monoid and the map $\operatorname{dim}$ is a monoid homomorphism, that is, for $q, r \in Q$,

$$
\operatorname{dim}(q r)=\operatorname{dim}(q) \operatorname{dim}(r),
$$

and

(iii) the product distributes over the addition in each fiber, that is, for $q, r_{1}, r_{2} \in Q$ with $\operatorname{dim}\left(r_{1}\right)=\operatorname{dim}\left(r_{2}\right)$,

$$
q\left(r_{1}+r_{2}\right)=q r_{1}+q r_{2},
$$

and the product associates with the product by scalars in the sense of

$$
\alpha(q r)=(\alpha q) r
$$

where $q, r \in Q$ and $\alpha \in F$.

The rank of $Q$ is the rank of its group of dimensions.

This structure can be thought of as an algebraic fiber bundle, where the base structure is the group $\mathscr{D}$ and where over each element of it we place a fiber which is a one dimensional vector space. If $A$ is a dimension and we denote $Q_{\mathrm{A}}=\operatorname{dim}^{-1}(\mathrm{~A})$ the fiber over it, then the space is made of the disjoint union of its fibers $Q=\cup_{\mathrm{A} \in \mathscr{D}} Q_{\mathrm{A}}$. Therefore, this structure is a particular instance of the structure presented in [3] as a pair dimension group - fibers: $\left\langle\mathscr{D},\left\{Q_{\mathrm{A}}\right\}\right\rangle$.

The fibers are not independent, for they have algebraic bounds given by the condition of the projection map being a monoid homomorphism. All fibers are isomorphic as vector spaces, and isomorphic to the field $F$, but there is one fiber of particular interest: the fiber $\operatorname{dim}^{-1}\left(1_{\mathscr{D}}\right)$, the set of quantities of dimension one. The identity element in $Q$ is denoted $1_{Q}$ and, since dim is a homomorphism, necessarily $\operatorname{dim}\left(1_{Q}\right)=1_{\mathscr{D}}$, so $1_{Q}$ is a quantity of dimension one, as expected. Therefore, the fiber $\operatorname{dim}^{-1}\left(1_{\mathscr{D}}\right)$ is not only an $F$ vector space, but an $F$-algebra of dimension 1 , that is, naturally isomorphic with the field $F$ by means of the isomorphism assigning the number 1 in $F$ with $1_{Q}$. For this reason, this fiber can be identified with $F$ when needed.

\subsection{System of units}

We now turn to the task of defining system of units. It has been noticed that a system of units is nothing but a choice of a nonzero quantity of each dimension, that is, a basis in each fiber. Remember that a system of units is called coherent if the product of the units of any two quantities $q$ and $r$ gives the unit in the system for the quantity $q r$. The tool for a precise definition is the concept of section, which is a map that chooses one, and only one, element in $Q$ from each fiber.

Definition 2.6. A section of the space of quantities $Q$ is a map $\sigma: \mathscr{D} \rightarrow Q$ such that $\operatorname{dim} \circ \sigma=\mathrm{id}_{\mathscr{D}}$. A section is called coherent if the map is a group homomorphism. The zero section, denoted $\sigma_{0}$, is the section which selects the zero element of each fiber. A nonzero section is a section none of which images is a zero element.

Then we have the following definition.
Definition 2.7. A system of units in a space of quantities is a nonzero section of it. The system is called coherent if the section is coherent.

Before proceeding further, a word on the zeros of $Q$ is necessary. Since each fiber has a zero element there are many zeros in the space $Q$, all of which constitute $\sigma_{0}(\mathscr{D})$, the image of the zero section. In this construction each zero has a dimension, so $0 \mathrm{~ms}^{-1}$ is a different quantity than $0 \mathrm{~kg}$.

Therefore, rather than speaking of the zero element, in this structure we have to speak of a zero element to refer ourselves to any of these elements in the image of the zero section. Despite of that, when no confusion is possible we write $q=0$ to symbolize that the quantity $q$ is a zero, without stating explicitly its dimension. Nevertheless, these zeros behave as is expected from an ordinary zero: the product of a quantity with a zero is a zero, as can be easily verified. However, it must be noticed that there is nothing in the definition of a space of quantities to prevent the existence of zero divisors, i.e. nonzero quantities $q$ and $r$ such that their product $q r$ is a zero. As an extreme example consider a space of quantities with a product defined as $q r=0$ for any dimensionful quantities $q$ and $r$; it satisfies all the axioms of definition 2.5. Zero divisors, if any, are by no means isolated for, if $q$ is a zero divisor, then $\alpha q$, with $\alpha \in F$ is also a zero divisor, so the entire fiber of $q$ is made of zero divisors. Also if $s$ is another quantity such that $s q$ is not zero, then $s q$ is another zero divisor. Of course zero divisors do not show up in spaces of quantities of actual measurements, therefore in what follows we only consider spaces of quantities free of zero divisors. Some advantages we gain from that are collected in the next proposition.

Proposition 2.8. In a space of quantities the following properties are equivalent:

\section{$i$. There are no zero divisors.}

ii. The set of nonzero quantities is a group with respect to the product.

\section{iii. There exists a coherent system of units.}

PROOF. We show the first property to be equivalent to each of the other two. In order to show that the set of nonzero quantities is a group we need to show it is closed under the product and every element has an inverse. The absence of zero divisors is just the former condition, so let us show the inverse of a nonzero quantity $q$. Let $\tilde{q}$ be a nonzero quantity in the inverse fiber of $q$, meaning $\operatorname{dim}(\tilde{q})=\operatorname{dim}(q)^{-1}$. Therefore, $q \tilde{q}$ is nonzero and dimensionless and, thus, there is a nonzero scalar $\alpha$ such that $q \tilde{q}=\alpha 1_{Q}$. The quantity $s=\alpha^{-1} \tilde{q}$ satisfies $q s=1_{Q}$. On the contrary, if there are zero divisors, the set of nonzero quantities is not closed under the product, so it is not a group.

Now assume again $Q$ is free of zero divisors and define a section $\sigma: \mathscr{D} \rightarrow Q$ by assigning a nonzero element in the corresponding fiber to each element in a basis of $\mathscr{D}$ and the rest 
of elements by asking $\sigma$ to be a homomorphism. The absence of zero divisors ensures that $\sigma$ is a nonzero, in addition to coherent, section. On the contrary, assume now that $q$ and $r$ are nonzero elements of $Q$ such that $q r$ is a zero, and let $\sigma$ be a coherent section of $Q$. Assume $\sigma(\operatorname{dim}(q))$ and $\sigma(\operatorname{dim}(r))$ are nonzero. Then they are of the form $\sigma(\operatorname{dim}(q))=\alpha q$ and $\sigma(\operatorname{dim}(r))=\beta r$ for some nonzero $\alpha$ and $\beta$ in $F$. We have $\sigma(\operatorname{dim}(q r))=(\alpha q)(\beta r)=\alpha \beta q r$ which is a zero. Hence, a coherent section is not nonzero, so there exits no coherent system of units.

The following is an explicit construction of a space of quantities given a group of dimensions $\mathscr{D}$. Moreover, in section 4 we justify that, to some extent, this is the only example of a space of quantities free of zero divisors.

Example 2.9. For a field $F$ and a finitely generated free Abelian group $\mathscr{D}$, the set $F \times \mathscr{D}$ together with the map dim: $F \times \mathscr{D} \rightarrow \mathscr{D}$ which projects onto the second component, and the operations

$$
\begin{array}{r}
(\alpha, \mathrm{A})+(\beta, \mathrm{A})=(\alpha+\beta, \mathrm{A}), \\
\beta(\alpha, \mathrm{A})=(\beta \alpha, \mathrm{A}), \\
(\alpha, \mathrm{A})(\beta, \mathrm{B})=(\alpha \beta, \mathrm{AB}),
\end{array}
$$

for $\alpha, \beta \in F$ and $\mathrm{A}, \mathrm{B} \in \mathscr{D}$, becomes a space of quantities free of zero divisors. A coherent system of units is given from a group homomorphism $\chi: \mathscr{D} \rightarrow F^{*}$, where $F^{*}$ denotes the multiplicative group of the field, by $\sigma: \mathscr{D} \rightarrow F \times \mathscr{D}: \mathrm{A} \mapsto$ $(\chi(\mathrm{A}), \mathrm{A})$.

The first goal of a formalization of quantity calculus is to justify within the formalism the actual way in which operations between quantities are performed, that is, with the aid of a system of units and operating with the numerical values and with the units separately. The following paragraphs do this. Let us start by writing down the expression of any quantity in Maxwell's form. Let $q$ be a quantity in a space $Q$ free of zero divisors, and let $\sigma$ be a system of units in $Q$. The dimension of $q$ is $\operatorname{dim}(q)$ and the unit in its fiber is $\sigma(\operatorname{dim}(q))$. Now, since the latter is not a zero, by proposition 2.8 it has an inverse so we can define the map $v: Q \rightarrow F$, in which we make use of the identification of the field $F$ with the fiber $\operatorname{dim}^{-1}\left(1_{\mathscr{D}}\right)$ of quantities of dimension one, by

$$
v(q)=q \sigma(\operatorname{dim}(q))^{-1} .
$$

The quantities $q$ and $\sigma(\operatorname{dim}(q))$ have the same dimension, so the product in equation (4) gives a quantity of dimension one which, after identification with an element of $F$, can be regarded as a number: the numerical value of $q$ with respect to the unit $\sigma(\operatorname{dim}(q))$. Then we have

$$
q=v(q) \sigma(\operatorname{dim}(q)),
$$

where we identify $v(q)$ with $\{q\}$ and $\sigma(\operatorname{dim}(q))$ with $[q]$ as given in equation (1). In other words, the symbols $\{\cdot\}$ and $[\cdot]$ are nothing but the maps $\{\cdot\}=v$ and $[\cdot]=\sigma \circ \operatorname{dim}$. Let us study their algebraic properties.
Proposition 2.10. In a space of quantities free of zero divisors, the map $[\cdot]: Q \rightarrow Q$ verifies

(i) for $q_{1}, q_{2}$ quantities in the same fiber, and $\alpha, \beta$ in $F$

$$
\left[\alpha q_{1}+\beta q_{2}\right]=\left[q_{1}\right]=\left[q_{2}\right]
$$

(ii) it is a homomorphism with respect to the product of quantities if and only if $\sigma$ is a coherent section.

PROOF. Both items stem directly from the splitting of $[\cdot]$ as the composition $\sigma \circ \mathrm{dim}$. For the first one, since $q_{1}$ and $q_{2}$ are in the same fiber, then so is $\alpha q_{1}+\beta q_{2}$, so it is clear that $\operatorname{dim}\left(\alpha q_{1}+\beta q_{2}\right)=\operatorname{dim}\left(q_{1}\right)=\operatorname{dim}\left(q_{2}\right)$ and, therefore, the same applies to the map [.]. For the second item, if $\sigma$ is a group homomorphism, then [.] is the composition of two homomorphisms with respect to the product, so it is also a homomorphism. For the other way around, if [.] is a homomorphism, so is $\sigma$ because the map dim is surjective.

Proposition 2.11. In a space of quantities free of zero divisors, the map $\{\cdot\}=v$ defined by equation (4) is

(i) an F-linear homomorphism and

(ii) a homomorphism with respect to the product of quantities if and only if $\sigma$ is a coherent section.

Proof. For the first item consider two quantities $q_{1}$ and $q_{2}$ in the same fiber and two scalars $\alpha$ and $\beta$ in the field $F$ and compute $\left\{\alpha q_{1}+\beta q_{2}\right\}=\left(\alpha q_{1}+\beta q_{2}\right) \sigma\left(\operatorname{dim}\left(\alpha q_{1}+\right.\right.$ $\left.\left.\beta q_{2}\right)\right)^{-1}$. Since $\operatorname{dim}\left(\alpha q_{1}+\beta q_{2}\right)=\operatorname{dim}\left(q_{1}\right)=\operatorname{dim}\left(q_{2}\right)$ as in the previous proposition, the former expression can be written as $\alpha q_{1} \sigma\left(\operatorname{dim}\left(q_{1}\right)\right)^{-1}+\beta q_{2} \sigma\left(\operatorname{dim}\left(q_{2}\right)\right)^{-1}$, that is, $\alpha\left\{q_{1}\right\}+\beta\left\{q_{2}\right\}$.

In the second item the if part is trivial. For the only if part consider $\mathrm{A}$ and $\mathrm{B}$ in $\mathscr{D}$ and choose two nonzero quantities $q$ and $r$ such that $\mathrm{A}=\operatorname{dim}(q)$ and $\mathrm{B}=\operatorname{dim}(r)$. Then $\sigma(\mathrm{AB})=$ $q r\{q r\}^{-1}$, since $q r$ is not a zero, then $\{q r\} \neq 0$. Now, because $\{\cdot\}$ is a homomorphism with respect to the product, the previous expresion gives $q\{q\}^{-1} r\{r\}^{-1}=\sigma(\mathrm{A}) \sigma(\mathrm{B})$.

These propositions set the condition to operate with quantities in the usual way: for quantities $q_{1}$ and $q_{2}$ in the same fiber and scalars $\alpha$ and $\beta$

$\left\{\alpha q_{1}+\beta q_{2}\right\}=\alpha\left\{q_{1}\right\}+\beta\left\{q_{2}\right\} ; \quad\left[\alpha q_{1}+\beta q_{2}\right]=\left[q_{1}\right]=\left[q_{2}\right]$,

and for any quantities $q$ and $r$, only in case of a coherent system of units,

$$
\{q r\}=\{q\}\{r\} ; \quad[q r]=[q][r] .
$$

In other words, the equations (2) have been justified from the axioms. 


\section{NEW SPACES FROM OLD ONES}

\subsection{Subspace}

Definition 3.1. A subset $S$ of a space of quantities $Q$ is a subspace if, with the operations of $Q$ and the restriction of the projection map, it is a space of quantities.

Since the projection map dim restricted to $S$ is the projection map of $S$, its image, $\operatorname{dim}(S)$, must be a subgroup of $\mathscr{D}$. Fortunately, it is a well known result of group theory that a subgroup of a free Abelian group is itself free Abelian [26, Theorem 10.17]. This is equivalent to the condition of $S$ being closed under the product of quantities. In particular, $1_{\mathscr{D}}$ is in this subgroup. The subset $S$ is also closed under addition of quantities of the same fiber and product by scalars so, if $s$ is a nonzero quantity in $S$, then $\alpha s$, for any scalar $\alpha$, is also in $S$. In other words, the complete fiber containing $s$ is in $S$, as should be, and for the fibers in $S$ must be one dimensional vector spaces. This observation rules out a fiber in $S$ containing only the zero element. As a consequence, the fiber of dimensionless quantities is contained in $S$. Thus, we have characterized the subspaces of a space of quantities as follows.

Proposition 3.2. Let $Q$ be a space of quantities with projection map dim: $Q \rightarrow \mathscr{D}$. A subset $S \subset Q$ is a subspace if and only if it is of the form $S=\operatorname{dim}^{-1}(\mathscr{E})$, where $\mathscr{E}$ is a subgroup of the group of dimensions $\mathscr{D}$.

Trivially $Q=\operatorname{dim}^{-1}(\mathscr{D})$ is a subspace, arising from the improper subgroup of $\mathscr{D}$, and so is the fiber $\operatorname{dim}^{-1}\left(1_{\mathscr{D}}\right)$, the subspace arising from the trivial subgroup of $\mathscr{D}$. Some nontrivial examples follow.

Example 3.3. The space $Q_{\text {geom }}$ can be seen is a subspace of $Q_{\text {kin }}$, for $Q_{\text {geom }}=\operatorname{dim}^{-1}(\langle\mathrm{~L}\rangle)$, and $\langle\mathrm{L}\rangle$ is a subgroup of $\mathscr{D}_{\text {kin }}$. Analogously, $Q_{\text {kin }}$ as a subspace of $Q_{\mathrm{mech}}$, which in turn is a subspace of $Q_{\mathrm{phys}}$.

But also $\operatorname{dim}^{-1}\left(\left\langle\mathrm{~L}^{2}\right\rangle\right)$ is a subspace of $Q_{\mathrm{geom}}$ with group of dimensions $\left\langle\mathrm{L}^{2}\right\rangle$.

\subsection{Tensor product}

From the examples one intuitively expects to be able to build the space $Q_{\text {kin }}$ of kinematics quantities from $Q_{\text {geom }}$ and $Q_{\text {time }}$, the spaces of quantities of geometry and time, respectively. But a simple cartesian product is not enough, for running 10 meters in 1 second gives the same speed as running 100 meters in 10 seconds. The technique is somewhat similar to the tensor product of linear spaces so we adopt the notation. Let $Q$ and $R$ be spaces of quantities free of zero divisors, over the field $F$ and with groups of dimensions $\mathscr{D}_{Q}$ and $\mathscr{D}_{R}$, respectively, and projection maps $\operatorname{dim}_{Q}$ and $\operatorname{dim}_{R}$.

In the set $Q \times R$ define the element $\left(q_{1}, r_{1}\right)$ to be related to $\left(q_{2}, r_{2}\right)$ if there is $\alpha \in F$ such that $q_{1}=\alpha q_{2}$ and $r_{2}=\alpha r_{1}$ or such that $q_{2}=\alpha q_{1}$ and $r_{1}=\alpha r_{2}$. It is straightforward to check it is an equivalence relation. The quotient set is denoted $Q \otimes R$ and the equivalence class of the element $(q, r)$ is denoted $q \otimes r$. Notice $\alpha q \otimes r=q \otimes \alpha r$, in particular $q \otimes 0=0 \otimes 0=0 \otimes r$.
We now define a structure of space of quantities in $Q \otimes$ $R$. Its group of dimensions is the direct product of $\mathscr{D}_{Q}$ and $\mathscr{D}_{R}$, which is a free Abelian group with rank the sum of the ranks of each one. The projection map is defined by $\operatorname{dim}(q \otimes$ $r)=\left(\operatorname{dim}_{Q}(q), \operatorname{dim}_{R}(r)\right)$, which is well defined because all the elements in the class $q \otimes r$ have the same image under dim. Define a product in $Q \otimes R$ by $\left(q_{1} \otimes r_{1}\right)\left(q_{2} \otimes r_{2}\right)=\left(q_{1} q_{2}\right) \otimes$ $\left(r_{1} r_{2}\right)$, which is independent of the representatives chosen, is commutative and associative and has an identity element: the class $1_{Q} \otimes 1_{R}$.

Define the product of the scalar $\gamma \in F$ times $q \otimes r$ by $\gamma(q \otimes r)=(\gamma q) \otimes r=q \otimes(\gamma r)$. Finally, define the addition of two elements in the same fiber $q_{1} \otimes r_{1}$ and $q_{2} \otimes r_{2}$ in the following manner. From the absence of zero divisors and proposition 2.8 there are nonzero $q \in Q$ and $r \in R$ such that $q_{i}=\alpha_{i} q$ and $r_{i}=\beta_{i} r$ for some $\alpha_{i}, \beta_{i} \in F, i \in\{1,2\}$; define $q_{1} \otimes r_{1}+q_{2} \otimes r_{2}=\left(\alpha_{1} \beta_{1}+\alpha_{2} \beta_{2}\right)(q \otimes r)$. The addition and product by scalars in the set of elements of a fiber satisfy the properties of a vector space over $F$ and, moreover, this vector space is of dimension one, for, if $q$ and $r$ are nonzero elements and $\alpha$ and $\beta$ are arbitrary scalars, then $(\alpha q) \otimes(\beta r)=(\alpha \beta)(q \otimes r)$. The zero element in each fiber is $0 \otimes 0$.

Finally, it is also straightforward to see the projection map behaves well under the product: $\operatorname{dim}\left(\left(q_{1} \otimes r_{1}\right)\left(q_{2} \otimes r_{2}\right)\right)=$ $\operatorname{dim}\left(q_{1} \otimes r_{1}\right) \operatorname{dim}\left(q_{2} \otimes r_{2}\right)$. Then we have the following result.

Proposition 3.4. The set $Q \otimes R$, together with the operations defined above, is a space of quantities over the field $F$ with group of dimensions $\mathscr{D}_{Q} \times \mathscr{D}_{R}$ and rank $\operatorname{rank}(Q)+\operatorname{rank}(R)$.

The spaces $Q$ and $R$ can be identified, respectively, with $Q \otimes \operatorname{dim}_{R}^{-1}\left(1_{\mathscr{D}_{R}}\right)$ and $\operatorname{dim}_{Q}^{-1}\left(1_{\mathscr{D}_{Q}}\right) \otimes R$, which are subspaces of $Q \otimes R$.

Example 3.5. As announced before, we have $Q_{\text {kin }} \cong Q_{\text {geom }} \otimes$ $Q_{\text {time. }}$. Also $Q_{\text {mech }} \cong Q_{\text {kin }} \otimes Q_{\text {mass }}$.

\subsection{Quotient space}

The quotient space is a construction intended to reduce the rank of a space of quantities by identifying certain quantities of different dimensions. The quotient cannot be taken with respect to a subspace, but another kind of subset of $Q$, namely, that given by a subsection.

Definition 3.6. Let $Q$ be a space of quantities free of zero divisors. A subsection of $Q$ is the restriction of a nonzero coherent section $\sigma: \mathscr{D} \rightarrow Q$ to a subgroup $\mathscr{E}$ of $\mathscr{D}$.

Its image $\Sigma=\sigma(\mathscr{E})$, which is also called subsection for brevity, is the intersection of the subspace $\operatorname{dim}^{-1}(\mathscr{E})$ and $\sigma(\mathscr{D})$, the image of the section. Notice that $1_{Q} \in \Sigma$ because the section $\sigma$ is coherent. With the aid of $\Sigma$ we can define an equivalence relation in $Q$. The quantity $q_{2}$ is equivalent modulo $\Sigma$ to the quantity $q_{1}$ if $q_{2}=q_{1} s$ for some quantity $s$ in $\Sigma$. It is reflexive for, as noticed before, $1_{Q} \in \Sigma$. It is symmetric, because $s$ is not a zero and, by the absence of zero divisors, it is invertible in $Q$ and $q_{1}=q_{2} s^{-1}$, where $s^{-1}$ is in $\Sigma$ because 
the section is coherent. Finally, if $q_{2}=q_{1} s$ and $q_{3}=q_{2} s^{\prime}$ for $s$ and $s^{\prime}$ in $\Sigma$ then $q_{3}=q_{1} s s^{\prime}$, so $q_{3}$ is equivalent modulo $\Sigma$ to $q_{1}$ since $s s^{\prime}$ is in $\Sigma$ by the coherence of $\sigma$.

The quotient set of this equivalence relation is denoted $Q / \Sigma$ and its elements, the equivalence classes, are of the form $q \Sigma$, which denotes the set of the elements $q s$ with $s$ running in $\Sigma$. We now provide the quotient set with suitable operations to convert it into a space of quantities. First we describe its group of dimensions. Since equivalent elements $q_{1}$ and $q_{2}=$ $q_{1} s$ are identified in the quotient set, their dimensions must be identified as well. The obvious candidate for the group of dimensions is, thus, the quotient group $\mathscr{D} / \mathscr{E}$. In such a case it is only natural to define the projection map, $\widehat{\operatorname{dim}}: Q / \Sigma \rightarrow \mathscr{D} / \mathscr{E}$ by making the following diagram commutative.

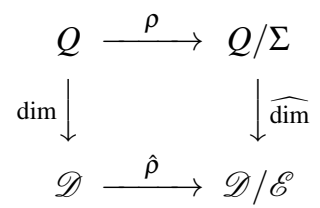

where the maps $\rho$ and $\hat{\rho}$ are the natural projections of each set into its respective quotient set. In other words, $\hat{\rho} \circ \operatorname{dim}=$ $\widehat{\operatorname{dim}} \circ \rho$. Unfortunately, the quotient of a free Abelian group is not necessarily free Abelian and, hence, $\mathscr{D} / \mathscr{E}$ does not necessarily qualify as a group of dimensions. Therefore, though the algebraic structure is well defined, the subsection must be carefully chosen so as the subgroup $\mathscr{E}$ makes the quotient $\mathscr{D} / \mathscr{E}$ a free Abelian group. For instance, in the group of dimensions of kinematics quantities, $\mathscr{D}_{\text {kin }} \cong\langle\mathrm{L}\rangle \times\langle\mathrm{T}\rangle$ the quotient $\mathscr{D} /\langle\mathrm{L}\rangle \cong\langle\mathrm{T}\rangle$ is free Abelian, while the quotient $\mathscr{D} /\left\langle\mathrm{L}^{2}\right\rangle \cong\langle\mathrm{L}\rangle /\left\langle\mathrm{L}^{2}\right\rangle \times\langle\mathrm{T}\rangle$ is not. From now on we assume that $\mathscr{E}$ is chosen so as to make $\mathscr{D} / \mathscr{E}$ free Abelian.

The product in $Q / \Sigma$ is defined by the rule $\left(q_{1} \Sigma\right)\left(q_{2} \Sigma\right)=$ $\left(q_{1} q_{2}\right) \Sigma$ which is easily checked to be independent of representatives. We have to check the condition which links the product and the projection map, but $\widehat{\operatorname{dim}}\left(q_{1} \Sigma q_{2} \Sigma\right)=$ $\widehat{\operatorname{dim}}\left(\left(q_{1} q_{2}\right) \Sigma\right)=\widehat{\operatorname{dim}} \circ \rho\left(q_{1} q_{2}\right)$ by the definition of the product in $Q / \Sigma$ and the definition of $\rho$. Now, by the commutativity of the diagram and because both, $\operatorname{dim}$ and $\hat{\rho}$, are homomorphisms, the latter expression equals $\hat{\rho} \circ \operatorname{dim}\left(q_{1} q_{2}\right)=$ $\hat{\rho} \circ \operatorname{dim}\left(q_{1}\right) \hat{\rho} \circ \operatorname{dim}\left(q_{2}\right)=\widehat{\operatorname{dim}}\left(q_{1} \Sigma\right) \widehat{\operatorname{dim}}\left(q_{2} \Sigma\right)$, so we conclude that $\widehat{\operatorname{dim}}$ is a monoid homomorphism.

The product with a scalar $\alpha$ from the field $F$ is defined by $\alpha(q \Sigma)=(\alpha q) \Sigma$, which is also independent of the choice of representative $q$ in the class $q \Sigma$. For the addition notice that if $q_{1} \Sigma$ and $q_{2} \Sigma$ are elements in the same fiber in $Q / \Sigma$, i.e. $\widehat{\operatorname{dim}}\left(q_{1} \Sigma\right)=\widehat{\operatorname{dim}}\left(q_{2} \Sigma\right)$, its sum cannot be defined simply as $\left(q_{1}+q_{2}\right) \Sigma$, because $q_{1}$ and $q_{2}$ need not be in the same fiber in $Q$. We only know $\operatorname{dim}\left(q_{2}\right)=\operatorname{dim}\left(q_{1}\right)$ A for some $\mathrm{A} \in$ $\mathscr{E}$. Denote $s=\sigma(\mathrm{A})$, an element in $\Sigma$, and define $q_{1}^{\prime}=q_{1} s$, so $q_{1} \Sigma=q_{1}^{\prime} \Sigma$, hence $\operatorname{dim}\left(q_{1}^{\prime}\right)=\operatorname{dim}\left(q_{1}\right) \operatorname{dim}(s)=\operatorname{dim}\left(q_{2}\right)$, so they are in the same fiber in $Q$. Now we can define the addition as $\left(q_{1} \Sigma\right)+\left(q_{2} \Sigma\right)=\left(q_{1}^{\prime}+q_{2}\right) \Sigma$. We could have taken instead an equivalent element of $q_{2}$ in the fiber of $q_{1}$ getting the same result. In the fiber of $q \Sigma$, the zero element is the class $q_{0} \Sigma$, where $q_{0}$ is the zero in the fiber of $q$, and is formed by the zeros of the fibers of $Q$ represented in the class $q \Sigma$.

It is straightforward to check that the conditions of definition 2.5 hold for $Q / \Sigma$, so we state the result as follows.

Proposition 3.7. If $\mathscr{E}$ is a subgroup of $\mathscr{D}$ such that $\mathscr{D} / \mathscr{E}$ is free Abelian then the set $Q / \Sigma$, together with the operations defined above, is a space of quantities with group of dimensions $\mathscr{D} / \mathscr{E}$ and rank given by $\operatorname{rank}(\mathscr{D})-\operatorname{rank}(\mathscr{E})$.

The mechanism of taking quotients is the algebraic tool underlying what is common practice in physics of choosing "systems of units" such that some specified universal constants become dimensionless and take on the numerical value 1. The extreme examples of this procedure are the systems of natural units, which do not cease to be a current subject of research as seen in [27]. But it has to be remarked that the mechanism goes beyond a change of system of units; it is indeed a change of space of quantities.

Example 3.8. A usual agreement in particle physics is to "choose units such that $c=\hbar=1$ ", where $c$ is the speed of light and $\hbar$ is the reduced Planck's constant, and all the quantities are measured in powers of units of energy. In fact, it is a reduction of the space of quantities of mechanics, of rank 3, to a space of rank 1, by the way of a suitable quotient.

Consider the dimensions of the quantities $c$, $\hbar$ and $a$ unit of energy, say the electronvolt, $\mathrm{eV}$ : $\operatorname{dim}(c)=\mathrm{LT}^{-1}, \operatorname{dim}(\hbar)=$ $\mathrm{L}^{2} \mathrm{~T}^{-1} \mathrm{M}$ and $\operatorname{dim}(\mathrm{eV})=\mathrm{L}^{2} \mathrm{~T}^{-2} \mathrm{M}$. They are independent in the group $\mathscr{D}_{\mathrm{mech}}$, as can be seen by solving the equation $\operatorname{dim}(c)^{m_{1}} \operatorname{dim}(\hbar)^{m_{2}} \operatorname{dim}(\mathrm{eV})^{m_{3}}=1_{\mathscr{D}}$, whose unique solution is $m_{1}=m_{2}=m_{3}=0$. Moreover, they can generate the group since the equation $\operatorname{dim}(c)^{m_{1}} \operatorname{dim}(\hbar)^{m_{2}} \operatorname{dim}(\mathrm{eV})^{m_{3}}=\mathrm{L}^{a} \mathrm{~T}^{b} \mathrm{M}^{c}$ in the group is translated as the following system of linear equations in $\mathbb{Z}$ :

$$
\begin{cases}m_{1}+2 m_{2}+2 m_{3} & =a \\ -m_{1}-m_{2}-2 m_{3} & =b \\ m_{2}+m_{3} & =c\end{cases}
$$

The matrix of coefficients is unimodular, so it is invertible and the system has a unique solution for any values $a, b, c$ in $\mathbb{Z}$. Therefore, we can write $\mathscr{D}_{\mathrm{mech}}=\langle\operatorname{dim}(c), \operatorname{dim}(\hbar), \operatorname{dim}(\mathrm{eV})\rangle$ and consider the subgroup $\mathscr{E}=\langle\operatorname{dim}(c), \operatorname{dim}(\hbar)\rangle$. Define a coherent section $\sigma: \mathscr{D}_{\text {mech }} \rightarrow Q_{\text {mech }}$ by its action on this basis:

$$
\begin{aligned}
& \sigma\left(\mathrm{LT}^{-1}\right)=c, \\
& \sigma\left(\mathrm{L}^{2} \mathrm{~T}^{-1} \mathrm{M}\right)=\hbar, \\
& \sigma\left(\mathrm{L}^{2} \mathrm{~T}^{-2} \mathrm{M}\right)=\mathrm{eV} .
\end{aligned}
$$

Now this section, when restricted to the subgroup $\mathscr{E}$, defines a subsection $\Sigma$. The quotient $Q_{\text {mech }} / \Sigma$ has group of dimensions $\mathscr{D}_{\text {mech }} / \mathscr{E} \cong\langle\operatorname{dim}(\mathrm{eV})\rangle$ and rank 1. The dimensionless quantities include $\hbar$ and $c$, which are identified with $1_{Q_{\text {mech }}}$, as desired, and the rest of quantities have dimensions of a power of energy. For instance, the dimension $\mathrm{L}=\operatorname{dim}(c) \operatorname{dim}(\hbar) \operatorname{dim}(\mathrm{eV})^{-1}$, when carried to the quotient group, is $\hat{\rho}(\mathrm{L})=\operatorname{dim}(\mathrm{eV})^{-1}$, so lengths are measured as inverse energies. 
4. HOMOMORPHISM OF SPACES OF QUANTITIES. ISOMORPHIC SPACES

In this section the tool for comparison of spaces of quantities is defined and its properties studied. The goal is the classification of spaces of quantities, which is achieved in theorem 2.

Definition 4.1. Let $Q$ and $R$ be spaces of quantities over the field $F$. A map $\psi: Q \rightarrow R$ is a homomorphism of spaces of quantities if

(i) for any two quantities $q_{1}, q_{2}$ in $Q$

$$
\psi\left(q_{1} q_{2}\right)=\psi\left(q_{1}\right) \psi\left(q_{2}\right)
$$

that is, it is a monoid homomorphism with respect to the product, and

(ii) if $q_{1}$ and $q_{2}$ are quantities in the same fiber of $Q$, then $\psi\left(q_{1}\right)$ and $\psi\left(q_{2}\right)$ are in the same fiber in $R$ and

$$
\psi\left(\alpha q_{1}+\beta q_{2}\right)=\alpha \psi\left(q_{1}\right)+\beta \psi\left(q_{2}\right),
$$

for $\alpha$ and $\beta$ in $F$, so $\psi$ is a linear map in each fiber.

The homomorphism $\psi$ induces a group homomorphism between the base groups, $\mathscr{D}_{Q}$ and $\mathscr{D}_{R}$. If $\operatorname{dim}_{Q}$ and $\operatorname{dim}_{R}$ are the respective projection maps, define the map $\phi: \mathscr{D}_{Q} \rightarrow \mathscr{D}_{R}$ so that the following diagram commutes,

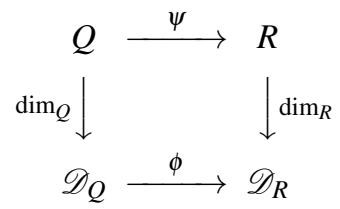

that is, $\operatorname{dim}_{R} \circ \psi=\phi \circ \operatorname{dim}_{Q}$. It is well defined because $\operatorname{dim}_{Q}$ and $\operatorname{dim}_{R}$ are surjective and $\psi$ preserves fibers and it is straightforward to check that $\phi$ is a group homomorphism.

The map $\phi$ says which fibers of $Q$ are mapped into each fiber of $R$. As an example, if $q$ is a quantity of dimension one in $Q$ then $\operatorname{dim}_{R}(\psi(q))=\phi\left(\operatorname{dim}_{Q}(q)\right)=\phi\left(1_{\mathscr{D}_{Q}}\right)=1_{\mathscr{D}_{R}}$, so the fiber of quantities of dimension one in $Q$ is mapped to the fiber of quantities of dimension one in $R$.

An isomorphism $Q \rightarrow R$ is a bijective homomorphism and defines $Q$ and $R$ as isomorphic spaces, denoted $Q \cong R$. The natural maps such as the identity $\operatorname{map} \operatorname{id}_{Q}: Q \rightarrow Q$, the inclusion map $i: Q \hookrightarrow R$, where $Q$ is a subspace of $R$, and the natural projection map $\rho: Q \rightarrow Q / \Sigma$, where $\Sigma$ is a subsection of $Q$, are all homomorphisms of spaces of quantities. The trivial homomorphism is the map which sends every element in $Q$ to the dimensionless zero of $R$. By a zero homomorphism we understand a homomorphism in which all the elements in $Q$ are mapped to zero elements in $R$, such as the trivial map, but there are other zero homomorphisms, as many as group homomorphisms between $\mathscr{D}_{Q}$ and $\mathscr{D}_{R}$. For given such a group homomorphism $\phi$, which in turn defines which fibers in $Q$ are mapped to which fibers in $R$, it is enough to define $\psi: Q \rightarrow R$ by sending each $q \in Q$ to the zero element of the fiber assigned by $\phi$.

In fact it is necessary to understand the behaviour of fibers and zeros under a homomorphism. It is clear that the image of a zero is a zero. If $q$ is a nonzero element of $Q$ but $\psi(q)$ is a zero in $R$, then all the fibers of $q$ are mapped to the same zero, for $\psi(\alpha q)=\alpha \psi(q)$ which is the same zero for any $\alpha \in F$. On the other hand, if $\psi(q)$ is not a zero, then the fiber of $q$ is mapped isomporphically (as vector spaces) to the fiber of $\psi(q)$. In particular, if $\psi\left(1_{Q}\right)$ is zero, then the homomorphism is a zero homomorphism, for $\psi(q)=\psi\left(q 1_{Q}\right)=\psi(q) \psi\left(1_{Q}\right)$ which is a zero for any $q$. This expresion also proves that if $\psi\left(1_{Q}\right)$ is not a zero then it is $1_{R}$, the identity in $R$.

So far a homomorphism can be defined by setting which fiber of $R$ is the image of each fiber of $Q$ and by setting which fibers of $Q$ are mapped to zero and which of them are mapped isomorphically to their corresponding fibers. In the case of interest of spaces free of zero divisors the result can be improved.

Proposition 4.2. Let $Q$ be a space of quantities free of zero divisors and $\psi: Q \rightarrow R$ a homomorphism of spaces of quantities. Then $\psi\left(1_{Q}\right)=0$ implies $\psi$ is a zero homomorphism, while $\psi\left(1_{Q}\right) \neq 0$ implies that each fiber is mapped isomorphically onto a fiber in $R$.

PROOF. The first part has already been proved. Assume now that $\psi\left(1_{Q}\right)$ is not a zero and let $q$ be a nonzero element of $Q$ which, thus, has an inverse $q^{-1}$. Since $\psi\left(1_{Q}\right)=\psi\left(q q^{-1}\right)=$ $\psi(q) \psi\left(q^{-1}\right)$ is not a zero, we conclude that $\psi(q)$ is not a zero. Since its fiber is a one dimensional vector space, the latter says that the fiber of $q$ is mapped isomorphically to the fiber $\psi(q)$.

Of course, only the nonzero homomorphisms are of interest for us to be able to compare spaces of quantities, so from now on we only consider this kind of homomorphisms. The following are basic properties of homomorphisms of spaces of quantities.

Proposition 4.3. Let $\psi: Q \rightarrow R$ be a nonzero homomorphism of spaces of quantities. Then:

(i) the image of a subspace of $Q$ is a subspace of $R$,

(ii) the preimage of a subspace of $R$ is a subspace of $Q$,

(iii) the preimage of a section of $R$ is a section of $Q$ and

(iv) the preimage of a subsection of $R$ is a subsection of $Q$.

PROOF. Let $S$ be a subspace of $Q$, which is characterized, by proposition 3.2, as $S=\operatorname{dim}_{Q}^{-1}(\mathscr{E})$ for a subgroup $\mathscr{E}$ of $\mathscr{D}_{Q}$. The projection of its image is $\operatorname{dim}_{R}(\psi(S))=\phi\left(\operatorname{dim}_{Q}(S)\right)=$ $\phi(\mathscr{E})$, which is a subgroup of $\mathscr{D}_{R}$. Since $\psi$ is a nonzero homomorphism, every fiber in $S$ is mapped onto a fiber in $\psi(S)$, so we conclude that $\psi(S)$ coincides with $\operatorname{dim}_{R}^{-1}(\phi(\mathscr{E}))$, so it is a subspace of $R$.

Consider now $S$ to be a subspace of $R$. Its inverse image $\psi^{-1}(S)$ is made of the fibers which are mapped into $S$. But these fibers are given by $\operatorname{dim}_{Q}^{-1}\left(\phi^{-1}\left(\operatorname{dim}_{R}(S)\right)\right)$. Since $\operatorname{dim}_{R}(S)$ is a subgroup of $\mathscr{D}_{R}$, so is $\phi^{-1}\left(\operatorname{dim}_{R}(S)\right)$ with respect to $\mathscr{D}_{Q}$ and, thus, $\psi^{-1}(S)$ is a subspace of $Q$. 
Let $\sigma$ be a section of $R$. Since all fibers of $R$ are represented in the section, it is clear that its inverse image, $\psi^{-1}\left(\sigma\left(\mathscr{D}_{R}\right)\right)$, contains at least an element from each fiber in $Q$. We now show that there is no more than one from each fiber. Assume $q_{1}$ and $q_{2}$ are elements in the same fiber in $Q$ with $\psi\left(q_{1}\right)$ and $\psi\left(q_{2}\right)$ in the section of $R$. Then $\psi\left(q_{1}\right)$ and $\psi\left(q_{2}\right)$ belong to the same fiber in $R$, which means $\psi\left(q_{1}\right)=\psi\left(q_{2}\right)$ because they are in a section. Therefore, since fibers in $Q$ are mapped isomorphically to fibers in $R$, this leads to $q_{1}=q_{2}$.

Finally, considering a subsection as the intersection of a subspace and a section in $R$ it is clear that the inverse image of such intersection is a intersection of a subspace and a section in $Q$, thus, a subsection.

The kernel of a nonzero homomorphism $\psi: Q \rightarrow R$ is defined as $\operatorname{ker} \psi=\psi^{-1}\left(1_{R}\right)$. Since $\left\{1_{R}\right\}$ is a subsection of $R$, its inverse image defines, by proposition 4.3 , a subsection in $Q$. The image of a homomorphism, $\operatorname{im} \psi=\psi(Q)$ is, by the same proposition, a subspace of $R$. The kernel and the image so defined satisfy an isomorphism theorem.

Theorem 1. Let $\psi: Q \rightarrow R$ be a nonzero homomorphism of spaces of quantities. Then

$$
Q / \operatorname{ker} \psi \cong \operatorname{im} \psi
$$

as spaces of quantities.

ProOF. The first step is to check that the quotient of the theorem is indeed a space of quantities. Let us denote by $K$ the kernel of $\psi$, which is a subsection of $Q$. Thus, we only have to show that the group of dimensions of $Q / K$ is free Abelian and, to that end, we have to identify the projection of $K$ on $\mathscr{D}_{Q}$. We claim this projection to be precisely the kernel of the induced group homomorphism: $\operatorname{dim}_{Q}(K)=\operatorname{ker} \phi$. Let $q$ be in $K$. Then $\phi \circ \operatorname{dim}_{Q}(q)=\operatorname{dim}_{R} \circ \psi(q)=1_{\mathscr{D}_{R}}$, so $\operatorname{dim}_{Q}(q) \in \operatorname{ker} \phi$ which shows one inclusion. Now let A be in $\operatorname{ker} \phi$, and let $q$ be a nonzero element in the fiber $\operatorname{dim}_{Q}^{-1}(\mathrm{~A})$. Then $1_{\mathscr{D}_{R}}=\phi(\mathrm{A})=\operatorname{dim}_{R} \circ \psi(q)$, so $\psi(q)$ has dimension one and can be written as $\psi(q)=\alpha 1_{R}$ for a nonzero $\alpha$ in $F$ (since $\psi$ is a nonzero homomorphism). Consider the element $q_{1}=\alpha^{-1} q$. Then $\psi\left(q_{1}\right)=1_{R}$, so $q_{1} \in K$ and $\operatorname{dim}_{Q}\left(q_{1}\right)=\operatorname{dim}_{Q}(q)=\mathrm{A}$, so $\mathrm{A} \in \operatorname{dim}_{Q}(K)$, which shows the other inclusion and the claim is proved. The group of dimensions of $Q / K$ is, thus, $\mathscr{D}_{R} / \operatorname{ker} \phi$, which is isomorphic with $\operatorname{im} \phi$ by the isomorphism theorem for groups. Since $\operatorname{im} \phi$ is a subgroup of $\mathscr{D}_{R}$ it is free Abelian, and so is $\mathscr{D}_{R} / \operatorname{ker} \phi$. Therefore, $Q / K$ is a space of quantities.

The rest of the proof is standard. Define the map $\hat{\psi}$ : $Q / K \rightarrow \operatorname{im} \psi$ by $\hat{\psi}(q K)=\psi(q)$. It is straightforward to check, first, it is well defined; second, it is a homomorphism of spaces of quantities; third, it is a bijection.

Example 4.4. Let us revisit example 3.8 from the viewpoint of homomorphisms. Consider the map $\psi: Q_{\text {mech }} \rightarrow Q_{\text {energy }}$ given in the following form: the image of the quantity $\mathrm{eV}$ be itself, while the image of the speed of light, $c$, be $1_{Q_{\text {energy }}}$ as well as the image of the reduced Planck's constant, $\hbar$. Then $\operatorname{ker} \psi=\left\{c^{n}: n \in \mathbb{Z}\right\} \cup\left\{\hbar^{n}: n \in \mathbb{Z}\right\}$ and $\operatorname{im} \psi=Q_{\text {energy. }}$ Theorem 1 says that $Q_{\text {mech }} / \operatorname{ker} \psi$ is isomorphic with $Q_{\text {energy. }}$

The next result is the classification theorem for spaces of quantities free of zero divisors.

Theorem 2. Two spaces of quantities over the same field, free of zero divisors, are isomorphic if and only if they have the same rank.

PROOF. First consider two spaces of quantities $Q \cong R$. Then there is an isomorphism $\psi: Q \rightarrow R$ which induces a group homomorphism $\phi: \mathscr{D}_{Q} \rightarrow \mathscr{D}_{R}$. We only need to show the latter to be an isomorphism for it is a well known result of the theory of free Abelian groups that two such groups are isomorphic if and only if they have the same rank [26, Theorem 10.14]. But that is obvious since the map $\phi$ is nothing but the rule which says which fiber in $Q$ is mapped to what fiber in $R$ and, since $\psi$ is an isomorphism, this mapping of fibers is a bijection.

Second, assume $Q$ and $R$ are two spaces of quantites of the same rank, that is, their groups of dimensions $\mathscr{D}_{Q}$ and $\mathscr{D}_{R}$ have the same rank. Therefore, there is a group isomorphism $\phi: \mathscr{D}_{Q} \rightarrow \mathscr{D}_{R}$ and it defines a bijection of the fibers in $Q$ with the fibers in $R$. If we can assign a linear isomorphism between each pair of fibers, we are done. To that end it is enough to map a nonzero element of each fiber in $Q$ with a nonzero element of its corresponding fiber in $R$. Now, since both $Q$ and $R$ are free of zero divisors, by proposition 2.8 each of them has a coherent system of units, say $\sigma_{Q}$ and $\sigma_{R}$, respectively. Define a map $\psi: Q \rightarrow R$ by giving its action on the set $\sigma_{Q}\left(\mathscr{D}_{Q}\right)$ so that the following diagram commutes,

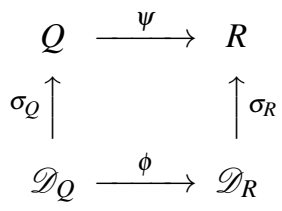

and extend it linearly in each fiber. This map is easily seen to be an isomorphism of spaces of quantities, so $Q \cong R$.

As a last example, we show that, up to isomorphism, the example 2.9 is the only space of quantities over a group of dimensions and a field free of zero divisors.

Example 4.5. Let $Q$ be a space of quantities over the field $F$ with group of dimensions $\mathscr{D}$ and free of zero divisors. Let $\sigma$ be a coherent system of units and $v$ the map defined in equation (4). Then the map $\psi: Q \rightarrow F \times \mathscr{D}$ given by $\psi(q)=$ $(v(q), \operatorname{dim}(q))$ is an isomorphism of spaces of quantities. Its inverse is $\psi^{-1}(\alpha, \mathrm{A})=\alpha \sigma(\mathrm{A})$.

This is to say that every space of quantities $Q$, free of zero divisors, is isomorphic with $F \times \mathscr{D}$. But the isomorphism is not canonical, for it depends on the system of units chosen. 


\section{CONCLUSIONS}

A brand-new algebraic structure has been defined and studied which fits exactly the algebra of quantity calculus. This structure is centered in the concept of dimension of a quantity as its main property, and considers only integer exponents in the combination of dimensions (and, thus, of units).

The axioms of definition 2.5 permit to deduce the usual expresion of a quantity as the product of a number times a unit, and to deduce the way the operations are usually performed. In addition, it has been shown how to construct new spaces from old ones by means of subspaces, tensor products or quotients. The latter construction is seen to be the correct interpretation of the usual procedure in physics, misleadingly referred to as a change of units, which sets some universal constant to 1 . Finally, a characterization and classification has been obtained in terms of the rank of the space of quantities, that is, the rank of its group of dimensions. This classification must be compared with that of Kitano [1], for they share some elements in common, but are not equivalent.

Kitano studies a set of physical quantities under different 'systems of units', even though these systems of units may have different number of basic units. In our approach, this should be considered as different spaces of quantities. Kitano then defines the relation $V \precsim U$ between the systems of units $V$ and $U$ if every pair of quantities $q$ and $p$ which are equal in $U$ are also equal in $V$ (equivalently, if every pair of distinguishable quantities in $V$ are also distinguishable in $U$ ). This relation is a preorder in the set of systems of units. In case $V \precsim U$ and $U \precsim V$ then $U$ and $V$ are said to be equivalent. If the system $U$ is supported by the algebraic structure $\mathbb{R} \times \mathbb{Q}^{n}$ and $V$ by $\mathbb{R} \times \mathbb{Q}^{m}$, then $V \precsim U$ implies $m \leq n$, and $U$ and $V$ can be equivalent only if $m=n$. But the contrary is not necessarily true, for there are systems of units with $m=n$ which are not comparable. An example of the latter is given by the electrostatic and the electromagnetic systems of units (esu and emu respectively). In the esu system the quantities $\mu_{0} / 4 \pi$ and $1 / c^{2}$, where $\mu_{0}$ is the permeability of empty space and $c$ is the speed of light, are not distinguisable, while $4 \pi \varepsilon_{0}$ is distinguishable from any of the former, being $\varepsilon_{0}$ the permittivity of empty space. In the emu system, however, $4 \pi \varepsilon_{0}$ is not distinguishable from $1 / c^{2}$, but $\mu_{0} / 4 \pi$ is; therefore, these systems are not comparable. It must be stressed that Kitano's classification is based on physical grounds, not algebraic ones, and thus is different from the one introduced in the present paper as we see in the next paragraph.

Within the context of the algebraic fiber bundle introduced in this paper, a similar preorder can be defined. We can write $Q \precsim R$ for two spaces of quantities $Q$ and $R$, over the same field $F$ and free of zero divisors, if there is a homomorphism $Q \rightarrow R$ which is an injection (i.e. a monomorphism). The injection property ensures that different quantities in $Q$ have different images in $R$. This is possible only if $\operatorname{rank} Q \leq \operatorname{rank} R$, as can be easily seen, and moreover, this condition is sufficient. Under this relation all spaces of quantities are comparable, and their comparison depends only on their ranks, in contrast with Kitano's classification. Resuming the previous example, the esu and emu systems can be understood as suitable quotients of the MKSA system. The MKSA system of units is defined in the space of quantities with group of dimensions $\mathscr{D}=\langle\mathrm{L}, \mathrm{T}, \mathrm{M}, \mathrm{I}\rangle$, of rank 4 . In this space, the quantity $1 / 4 \pi \varepsilon_{0}$ has dimension $\mathrm{L}^{3} \mathrm{~T}^{-4} \mathrm{MI}^{-2}$, while the dimension of the quantity $\mu_{0} / 4 \pi$ is $\mathrm{LT}^{-2} \mathrm{MI}^{-2}$. The esu system is obtained by making the quantity $1 / 4 \pi \varepsilon_{0}$ equal to 1 , that is, since the group $\mathscr{D}$ can be written as $\mathscr{D}=\left\langle\mathrm{L}, \mathrm{T}, \mathrm{I}, \mathrm{L}^{3} \mathrm{~T}^{-4} \mathrm{MI}^{-2}\right\rangle$, by taking the quotient of the space of quantities which gives the group of dimensions $\mathscr{D} / \mathscr{E}$, where $\mathscr{E}=\left\langle\mathrm{L}^{3} \mathrm{~T}^{-4} \mathrm{MI}^{-2}\right\rangle$, we thus, get a new space of quantities of rank 3 . The emu system, on the other hand, is obtained by making $\mu_{0} / 4 \pi$ equals 1 , that is, by the quotient in the group of dimensions $\mathscr{D} / \mathscr{F}$, where now $\mathscr{F}=\left\langle\mathrm{LT}^{-2} \mathrm{MI}^{-2}\right\rangle$, so it is also a new space of quantities of rank 3. According to theorem 2, these two spaces of quantities are isomorphic, regarded as algebraic structures, although Kitano's classification states them, righteously from a physical point of view, as incomparable. Nevertheless, it must be stressed that, under the algebraic theory depicted in this paper, the MKSA system and the esu (or emu) system are not different unit systems, but different spaces of quantities.

Finally, a comment on Domotor's torsor theory, which explains, in a series of three papers [28, 29, 4], in a convenient way, the passage from the state space of a physical measurement device to the space of quantities. His description of torsors is well suited to the algebraic structure of quantities given by Drobot and subsequent authors up to Kitano, but the same ideas may be adapted to the structure presented in this paper so as to provide the same foundation of the aforementioned passage. Indeed, the tool of torsors explains exactly, as he shows in his paper, the structure of the fibers, each of which is a vector space of dimension one.

\section{ACKNOWLEDGEMENT}

Thanks are due to the anonymous reviewer whose insightful and accurate comments have improved this paper.

\section{REFERENCES}

[1] Kitano, M. (2013). Mathematical structure of unit systems. Journal of Mathematical Physics, 54, 052901.

[2] Krystek, M. (2015). The term 'dimension' in the international system of units. Metrologia, 52, 297-300.

[3] Atkey, R., Ghani, N., Forsberg, F. N., Revell, T., Staton, S. (2015). Models for polymorphism over physical dimensions. In 13th International Conference on Typed Lambda Calculi and Applications (TLCA'15), 999-1013.

[4] Domotor, Z. (2017). Torsor theory of physical quantities and their measurement. Measurement Science Review, 17 (4), 152-177.

[5] Mills, I. (2016). On the units radian and cycle for the quantity plane angle. Metrologia, 53, 991-997.

[6] Mari, L., Giordani, A. (2012). Quantity and quantity value. Metrologia, 49, 756-764. 
[7] Feller, U. (2011). The International System of Unitsa case for reconsideration. Accreditation and Quality Assurance, 16, 143-153.

[8] Foster, M. (2010). The next 50 years of the SI: A review of the opportunities for the e-Science age. Metrologia, 47, R41-R51.

[9] Johansson, I. (2010). Metrological thinking needs the notions of parametric quantities, units and dimensions. Metrologia, 47, 219-230.

[10] Barrow, J.D., Gibbons, G.W. (2017). Maximum magnetic moment to angular momentum conjecture. Physical Review D ,95, 064040.

[11] Kostro, L. (2010). The physical meaning of the coefficients $c^{n} / G(n=1,2, \ldots 5)$ and the Standard Model of the Universe. AIP Conference Proceedings, 1316 (1), 165-179.

[12] Joint Committee for Guides in Metrology. (2012). International vocabulary of metrology - Basic and general concepts and associated terms (VIM). 3rd edition. JCGM 200:2012.

[13] de Boer, J. (1994). On the history of quantity calculus and the international system. Metrologia, 31, 405-429.

[14] Fourier, J.B.J. (1822). Théorie analytique de la Chaleur. Paris: Chez Firmin Didot, père et fils.

[15] Hallock, W., Wade, H.T. (1906). Outlines of the Evolution of Weights and Measures and the Metric System. The Macmillan Company.

[16] Maxwell, J. (1873). Treatise on Electricity and Magnetism. Oxford University Press.

[17] Buckingham, E. (1914). On physically similar systems: Illustrations of the use of dimensional equations. Physical Review, 4, 345-376.

[18] Landolt, M. (1945). Größe, Maßzahl und Einheit. Rascher Verlag Zurich. (French Translation - Paris: Dunod, 1947)

[19] Fleischmann, R. (1959/60). Einheiteninvariante Größengleichungen, Dimensionen. Der Mathematische und Naturwissenschaftliche Unterricht, 12, 385-443.

[20] Quade, W. (1961). Über die algebraische Struktur des Größenkalküls der Physik. Abhandlungen der Braunschweigischen Wissenschaftlichen Gesellschaft, 13, 2465. (Translated as an appendix to Dimensional Analysis for Economists by F.J. de Jong, Amsterdam: North Holland Publishing Company, 1967)

[21] Drobot, S. (1953). On the foundations of dimensional analysis. Studia Mathematica, 14, 84-99.

[22] Whitney, H. (1968). The mathematics of physical quantities: Part II: Quantity structures and dimensional analysis. The American Mathematical Monthly, 75 (3), 227256.

[23] Carlson, D. (1979). A mathematical theory of physical units, dimensions and measures. Archive for Rational Mechanics and Analysis, 70, 289-304.

[24] Giorgi, G. (1904). Proposals concerning electrical and physical units. In Transactions of the International Electrical Congress: St. Louis, 1904. Albany, N.Y.: J.B. Lyon Company, 136-141.

[25] Bridgman, P. (1922). Dimensional Analysis. Yale University Press.

[26] Rotman, J. (1995). An Introduction to the Theory of Groups, 4th edition. Springer.

[27] Lainscsek, C., Gorodnitsky, I. (2003). Planck's natural units are contained in a time series. AIP Conference Proceedings, 676 (1), 370-371.

[28] Domotor, Z. (2012). Algebraic frameworks for measurement in the natural sciencies. Measurement Science Review, 12 (6), 213-233.

[29] Domotor, Z. (2016). An algebraic approach to unital quantities and their measurement. Measurement Science Review, 16 (3), 103-126.

Received January 19, 2018. Accepted July 15, 2018. 